\title{
Cross-sectional Scanning Tunneling Microscopy and Spectroscopy of Compound Semiconductor Heterostructures
}

\author{
Shangjr GWO* and Hiroshi TOKUMOTO** \\ *Joint Research Center for Atom Technology-Angstrom Technology Partnership \\ (JRCAT-ATP), 1-1-4 Higashi, Tsukuba, Ibaraki, 305 Japan \\ **Joint Research Center for Atom Technology-National Institute for Aduanced \\ Interdisciplinary Research (JRCAT-NAIR), 1-1-4 Higashi, Tsukuba, Ibaraki, 305 Japan
}

(Received May 10, 1995, Accepted August 12, 1995)

\begin{abstract}
The advances in cross-sectional scanning tunneling microscopy and spectroscopy (XSTM/XSTS) are reviewed with emphasis on the structural and spectroscopic studies of semiconductor heterostructures on an atomic or nanometer scale. The unparalleled chemical and electronic sensitivities as well as spatial resolution of XSTM/XSTS are addressed. Examples shown here include recent results of cation/anion selective imaging, observations of individual impurities, interface roughness and asymmetry, band offset determination, and nanometer-resolution band mapping in prototype (AlGa)As/GaAs low-dimensional multilayered systems. Current developments in use of XSTM/XSTS combined with other techniques for the exploitation of optical properties of semiconductor heterostructures are also discussed.
\end{abstract}

\section{Introduction}

Remarkable advances in semiconductor growth technology have made possible the ability to tailor the electronic structure of materials through stacking of dissimiliar materials into multilayered heterostructures. Current state of the art growth techniques, such as molecular-beam epitaxy (MBE) and metalorganic chemical vapor deposition (MOCVD), can be used to achieve atomic-scale layer by layer growth and precise composition control of growing materials. These heterostructures are the building blocks for many advanced electronic and optoelectronic devices as well as future quantum devices.

Since the unusual properties of heterostructures originate from the formation of solid interfaces, a fundamentally important topic is the interplay between interfacial imperfection and electronic properties. In general, the interfaces are not perfact, i.e., not atomically flat over large distances and chemically abrupt. In fact, interfacial roughness and intermixing exist in most heterojunction

\footnotetext{
*e-mail address: gwo@jrcat.or.jp
}

devices. Therefore, the availability of a tool for real-space mapping of atomic and electronic structures across heterojunctions is crucial for a better understanding of heterostructure properties.

Scanning tunneling microscopy (STM), invented by Binnig and Rohrer in $1982,{ }^{1)}$ has created an entirely new way to probe the structure of semiconductor surfaces on an atomic scale. Based on STM operating principles, scanning tunneling spectroscopy (STS), which can be used to resolve local electronic properties of surfaces, was developed. Thus, it is very natural to extend the applicability of STM to study the properties of semiconductor interfaces. Recently, cross-sectional STM and STS (XSTM/XSTS) has been successfully applied to the interfacial studies of a number of important material systems including ( $\mathrm{AlGa}$ )As / GaAs, ${ }^{2,3)}$ (InGa)As/InP, ${ }^{4,5)}$ (InGa)As/GaAs, ${ }^{6}$ ) InAs/ GaSb, ${ }^{7,8)}$ (InGa)(AsP)/InP,${ }^{9)}$ and Si / SiGe. ${ }^{10)}$

There are two important issues in semiconductor heterojunctions that XSTM can be used to address: the crystallographic registry and the electronic structure on an atomic scale. Conventionally, the atomic ordering is monitored using reflection highenergy electron diffraction (RHEED) during epitaxial growth while cross-sectional transmission 
electron microscopy (XTEM) and X-ray diffraction (XRD) are used after the growth is completed. ${ }^{11,12)}$ The electronic properties semiconductor junctions are often investigated using analytical techniques such as photoluminescence, photoemission, or capacitance-voltage $(C-V)$ measurement. However, these techniques yield information which represents an average for many lattice unit cells within the crystal volume or for a large surface area as determined by the probing beam size.

On the other hand, XSTM is an extremely surface-sensitive tool which is capable of achieving atomic resolution. The results of a number of recent studies have demonstrated that STM and XSTM can be used to: (1) image a single atomic layer across heterojunctions, in contrast to the traditional XTEM method in which columnar averaging is used, (2) detect different chemical species, (3) resolve metallurgical imperfections such as alloy fluctuations and precipitates, and (4) gather electronic information resulting from intentional and unintentional structural imperfections such as doping impurities, point defects, and interface roughness. Thus, if we can prepare suitable cross-sectional surfaces of semiconductor heterojunctions, XSTM can yield structural, electronic, and in some cases, chemical information with unprecedented spatial resolution. And most importantly, it can provide insight into the correlation between structural and electronic properties.

Since STM is a surface-sensitive method, it is crucial that the bulk properties of heterojunctions are measurable at the cross-sectional surface. In the case of III-V compound semiconductor heterostructures, the heterostructures are typically grown on (001) substrates. Therefore, the atomically flat (110) cleavage plane naturally exposes the cross-sectional surface of heterostructures. It is well known that, on the (110) surface, the surface states lie outside of the fundamental band gap. As a result, the band-edge positions, as measured by tunneling spectroscopy, correspond directly to the band-edge positions within the bulk. Also, the (110) surface is a relaxed but unreconstructed $1 \times 1$ surface with empty states concentrated around group III atoms and filled states concentrated around group $\mathrm{V}$ atoms (a result of surface buckling). Thus, it is very easy to extract structural information for cation and anion sublattices independently from such a surface simply by reversing the polarity of applied voltage between sample and tip. For the above reasons, the XSTM/XSTS technique has recently attracted much attention. So far it has been applied mainly to III-V com- pound semiconductor systems as mentioned above. However, in principle, XSTM should also be applicable to the study of other heterostructure systems, such as those formed by group IV elements and II-VI compounds.

In this paper we review XSTM/XSTS studies on III-V compound semiconductors. In Sect. 2 we discuss important experimental considerations and techniques, which include an edge-finding algorithm, sample preparation, and equipment required to perform XSTM/XSTS. Following the experimental section, we show some recent XSTM results in Sect. 3. Most of the examples shown here focus on prototype two-dimensional and onedimensional (AlGa)As/GaAs systems because of their maturity in crystal growth and wide-spread use in various device applications. In Sect. 4, we show how the XSTS technique can be applied to study important electronic properties of heterointerfaces. Some recent developments for the investigation of optical properties of semiconductor heterostructures are briefly reviewed in Sect. 5 .

\section{Experimental Procedure}

The first obvious difficulty in performing XSTM is the in situ positioning of the tunneling tip within the epitaxial layer region which is usually less than a micron wide. This can be overcome by either using a UHV scanning electron microscope (SEM) ${ }^{13)}$ to help in positioning the tunneling tip within the epitaxial region or employing a high-resolution sample walker stage and an optical microscope. In the second approach (combination of a walker and an optical microscope), an edge-finding algorithm is usually employed: As shown in Fig. 1, after the sample is cleaved and transferred into the STM chamber, the tip is first positioned near the sample edge (the epitaxial region) with the aid of an optical microscope. The tip is then brought into the tunneling regime by the usual auto-approach procedure. Subsequent iteration of tip retraction, lateral stepp ing of the sample, and tip reapproaching is used to detect the edge of the sample (110) surface. This iteration process is terminated when the z-piezo voltage drastically exceeds (shown as an overshoot) the previous voltage at which tunneling occurred. The sample is then stepped laterally a small distance in the opposite direction to place the tip in a position appropriate for studying the epitaxial layer. The step size of the walker in the $\mathrm{x}$ direction can usually be calibrated by observing how many steps were required for the mirror image of the tip to move across the sample surface (the sample 


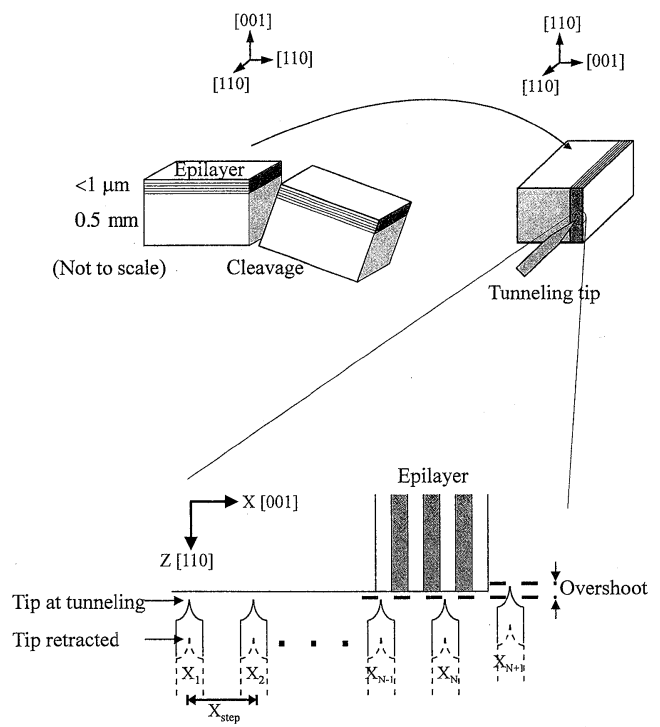

Fig. 1 Schematic diagram showing how the tip is positioned within the region of the epilayer in XSTM. [From Ref. 43)]

thickness is known). The success of this method depends primarily upon two things. First, the sample must be extremely flat all the way up to the edge. Second, a two-dimensional sample walker which is free of backlash and crosstalk is necessary. The first requirement is easily met by perfecting the cleaving technique. Usually, by thinning the substrate to below $100 \mu$, cross-sectional surfaces with excellent cleavage quality can be obtained by simply applying force to the prescribed epitaxial-layer surfaces. Currently, most of the STM sample (or tip) walkers used in XSTM studies are based on the stick-slip design. ${ }^{14-16)}$ To inhibit oxidation of the observed sample surfaces, a highgrade UHV environment and a high-resolution optical microscope (to speed the edge-finding process) are also highly recommended.

\section{XSTM of (AIGa)As/GaAs Heterostructures}

To date, due to the structural superiority and the availability of advanced growth techniques, a large portion of XSTM studies have been concentrated on the (AlGa)As / GaAs system. A number of interfacial and alloy properties of this system have been commonly accepted based on the massive amount of indirect experimental evidence which has been accumulated over the years. By using XSTM as a real-space probe, these properties can now be directly investigated on an atomic scale.

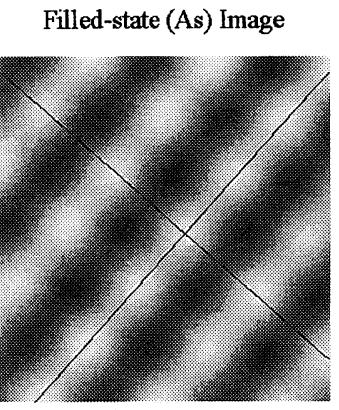

Empty-state (Ga) Image

Fig. 2 Dual-bias STM images of a GaAs(110) cleavage surface at negative (corresponding to filled-state image) and positive (corresponding to empty-state image) sample bias. The images were acquired at a sample bias voltage of $\pm 2.9 \mathrm{~V}$ and a tunneling current of $0.5 \mathrm{nA}$. The cross lines are used to indicate the same reference point in both imges.

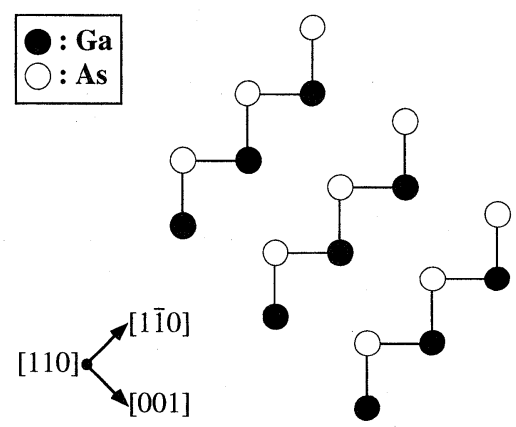

Fig. 3 The structure of the unreconstructed GaAs (110) cleavage surface.

Generally speaking, it is very difficult to obtain chemical composition information in STM/STM experiments. However, in the case of (110) crosssectional surfaces of III-V compound heterostructures, there are several electronically induced STM topographic contrasts, which result from differences in electronic characteristics rather than real-space differences. Therefore, by comparing intended growth structures with STM images, these contrasts can be used to obtain important chemical information. These electronically induced topographic contrasts can be classified according to their origins as follows: (1) charge transfer between cation and anion, (2) band edge position, (3) chemical bonding configuration, (4) impurity (e.g., dopants, anti-site defects). Cation-anion selective imaging was first demonstrated by Feenstra et al. ${ }^{17)}$ 
in the form of bias-polarity-dependent imaging of GaAs (110). On this surface the charge transfer from $\mathrm{Ga}$ to As results in filled states concentrated at the As sites and empty states concentrated at the $\mathrm{Ga}$ sites. As a result, in the constant-current topography (CCT) mode-tunneling current maintained constant through feedback control while rastering the tip across the sample surface-the positive-sample-bias (empty-state) image shows maximum topographic height at the $\mathrm{Ga}$ sites and the negative-sample-bias (filled-state) image shows maximum topographic height at the As sites. Shown in Fig. 2 is an example of dual-bias STM imaging of the unreconstructed GaAs (110) cleavage surface (Fig. 3 shows the atomic model).

The topographic contrast resulting from the difference in the band edge position can be due to the band discontinuities across heterojunctions or the different doping types and/or carrier concentrations. STM topographic contrast between two host materials of different band gaps in a heterostructure was first reported by Kato and coworkers. ${ }^{4,18-21)}$ in the InGaAs/InP type-I superlattice system (In a type-I semiconductor superlattice system, the band edges of the narrower-band-gap host material are located entirely within the band gap of the wider-band-gap host material. In a typeII semiconductor superlattice system, the top of the valence band of one of the host materials lies above the bottom of the conduction band of the other host material). ${ }^{22)}$ In a type-I system, when probing sample filled states, the available tunneling energy window is smaller in the wider band gap region at any fixed bias voltages between the sample and tip. Therefore, in the filled-state STM image of CCT mode, the wider band gap region (InP) will show darker topographic contrast than the narrower band gap material (InGaAs) since the tunneling tip has to come closer to the sample surface in order to reach the same tunneling current. The same kind of topography contrast has also been observed in other type-I heterostructure systems ((AlGa)As/GaAs, ${ }^{2,3)} \mathrm{SiGe} / \mathrm{Si},{ }^{10)}$. In contrast, in the type-II heterostructure system, the contrast between wider band gap and narrower band gap materials in the filled-state image is reversed. For example, in the filled-state topography of an In$\mathrm{As} / \mathrm{GaSb}$ superlattice, the InAs regions show a darker contrast than the GaSb regions. ${ }^{7,8,23)}$ The situation for the empty-state imaging turns out to be more complicated due to the existence of multiple-valley conduction band structure in the III-V compound semiconductors. In the GaAs or Si $p-n$ junctions, topographic contrast between regions of different doping types and/or carrier concentrations have also been reported. ${ }^{24-29)}$

The third type of electronically induced topographic contrast results from a difference in the bonding configuration of the imaged atoms. For example, in the case of a ternary random alloy such as (AlGa)As, each As atom is bonded to a different number of nearest-neighbor $\mathrm{Al}$ (or $\mathrm{Ga}$ ) atoms. Because the Al-As bond is more ionic than the $\mathrm{Ga}-\mathrm{As}$ bond, more $\mathrm{Al}$ nearest neighbors result in more charge transfer to the As atom. This changes the As-related surface state so that the tip approaches the surface more closely to maintain constant tunneling current in the filled-state tunneling. This feature turns out to be extremely useful for observing alloy composition fluctuations and alloy ordering on an atomic scale using STM. ${ }^{30,31)}$

Understanding the properties and distributions of various impurities in heterostructures is very important for appropriately controlling the desired electronic features of heterostructures. Since STM is extremely sensitive to local electronic variations, it is a natural choice for this purpose. Recent XSTM studies on the (110) cleavage surface of GaAs have indeed revealed that antisite point defects $\left(\mathrm{As}_{\mathrm{Ga}}\right)$ in low-temperaturegrown $\mathrm{GaAs}^{32,33)}$ and substitutional shallow donors and acceptors $\left(\mathrm{Si}_{\mathrm{Ga}}{ }^{34)} \mathrm{Zn}_{\mathrm{Ga}}{ }^{35,36)} \mathrm{Be}_{\mathrm{Ga}}{ }^{35)}\right)$ in doped GaAs can be imaged on an atomic scale. Moreover, since the potential perturbations originating from impurities usually extend over several lattice unit cells, the observable impuritiies are limited only to the topmost surface atomic plane. For example, due to the change of tunneling probability in the presence of an additional Coulomb potential near electrically active shallow donors/acceptors, the electrically active donors/acceptors are observed as bright protrusions of several different sizes superimposed on the atomically resolved lattice image. Shown in Fig. 4 is an example of a single $\mathrm{Si}_{\mathrm{Ga}}$ donor image obtained with a positive sample bias $(+2.0 \mathrm{~V})$ on a Sidoped GaAs sample $\left(1 \times 10^{18} \mathrm{~cm}^{-3}\right)$. By comparing topographic heights of different bright protrusions imaged with the same bias voltage, we can determine in which layers from the topmost surface plane the imaged impurities are located. In some cases of impurity imaging, characteristic shapes are also found for different types of impurities. ${ }^{32,36}$ This offers unique opportunities for performing chemical identification of impurities in these cases. However, special care should be taken to ensure that the observed characteristic shapes of impurities are reproducible and are not resulted from tip effects. 

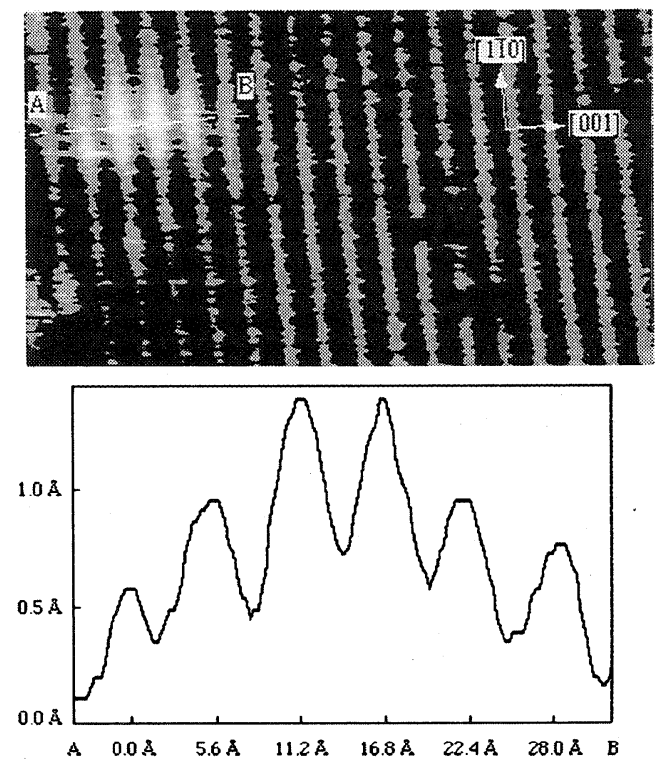

Fig. 4 STM empty-state image of a Si-doped $\left(1 \times 10^{18} \mathrm{~cm}^{-3}\right) \mathrm{GaAs}(110)$ cleavage surface. At the Si substitutional dopant site, we can see a bright surface protrusion superimposed on the background Ga-sublattice image. The height of this protrusion is shown in the corresponding line-cut displayed below the image. The image was acquired at a sample bias voltage of $+2.0 \mathrm{~V}$ and a tunneling current of $1.0 \mathrm{nA}$.

One very important aspect in the formation of heterointerfaces is the dependence of interface roughness on the sequence of layer growth. ${ }^{37)}$ In the (AlGa)As/GaAs system, the growth sequence has been found to play an important role in determining interface roughness. During the MBE growth process, due to the different migration lengths of the group III adatoms $(\mathrm{Ga} \approx 200 \AA$, $\mathrm{Al} \approx 35 \AA),{ }^{38)}$ the interface formed by (AlGa)As grown on GaAs ("normal" interface) is smoother than the interface formed by GaAs grown on (AlGa)As "inverted" interface). For minimizing the interface roughness, application of the growth interruption technique at each interface has been found to be very effective. In this approach, growth interruption allows the small terraces to relax into larger terraces through adatom diffusion. The duration of growth interruption usually varies from a few seconds to several minutes. The effects of growth interruption on the interface quality of (AlGa)As/GaAs heterojunctions and AlAs/GaAs short-period superlattices were recently studied by (a)

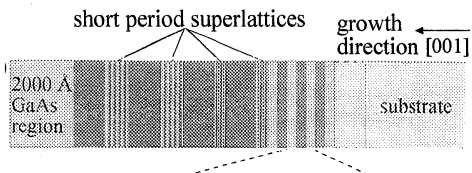

(b)

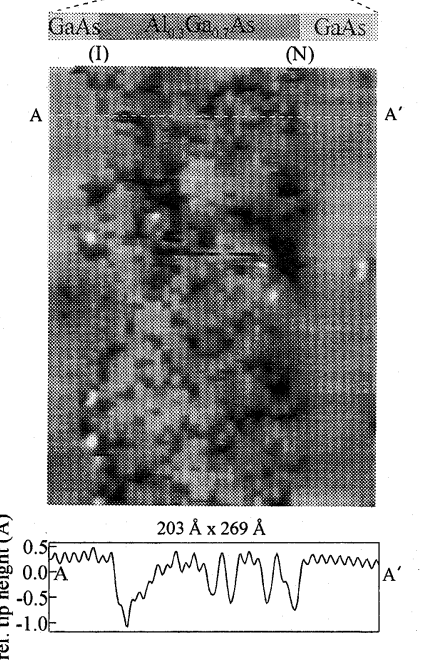

(c)
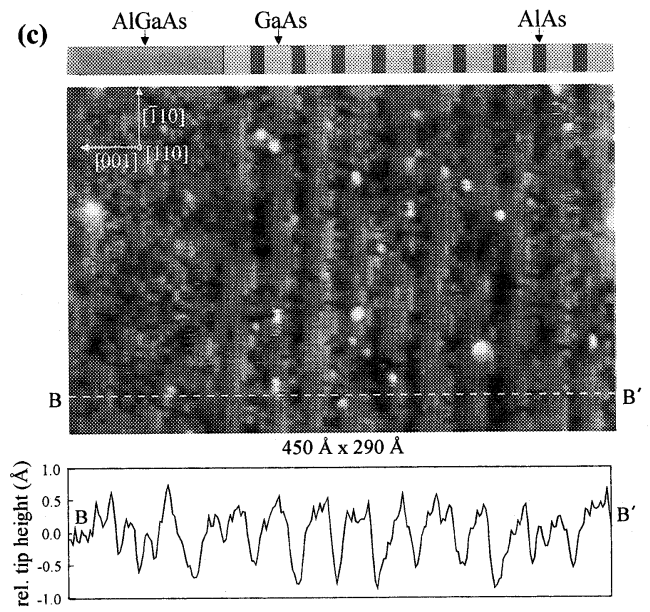

Fg. 5 (a) MBE growth sequence. (b) STM image of $\left(\mathrm{Al}_{0.3} \mathrm{Ga}_{0.7}\right) \mathrm{As} / \mathrm{GaAs}$ region. The sample bias was $-2.13 \mathrm{~V}$, and the tunneling current was $0.29 \mathrm{nA}$. The interface on the right (normal interface) appears sharper than the one on the left (inverted interface) by one to two atomic layers. This region was grown without growth interruption. The patchy feature in the (AlGa)As layer reflects local variations in AlAs content. (c) STM image of $(\mathrm{AlAs})_{2} /(\mathrm{GaAs})_{4}$ superlattice region acquired at a sample bias of $-2.25 \mathrm{~V}$ and a tunneling current of $0.2 \mathrm{nA}$. The apparent height difference between GaAs and AlAs is about $1 \AA$. The growth interruption time between AlAs and GaAs layers was $30 \mathrm{sec}-$ onds. [From Ref. 39)]. 


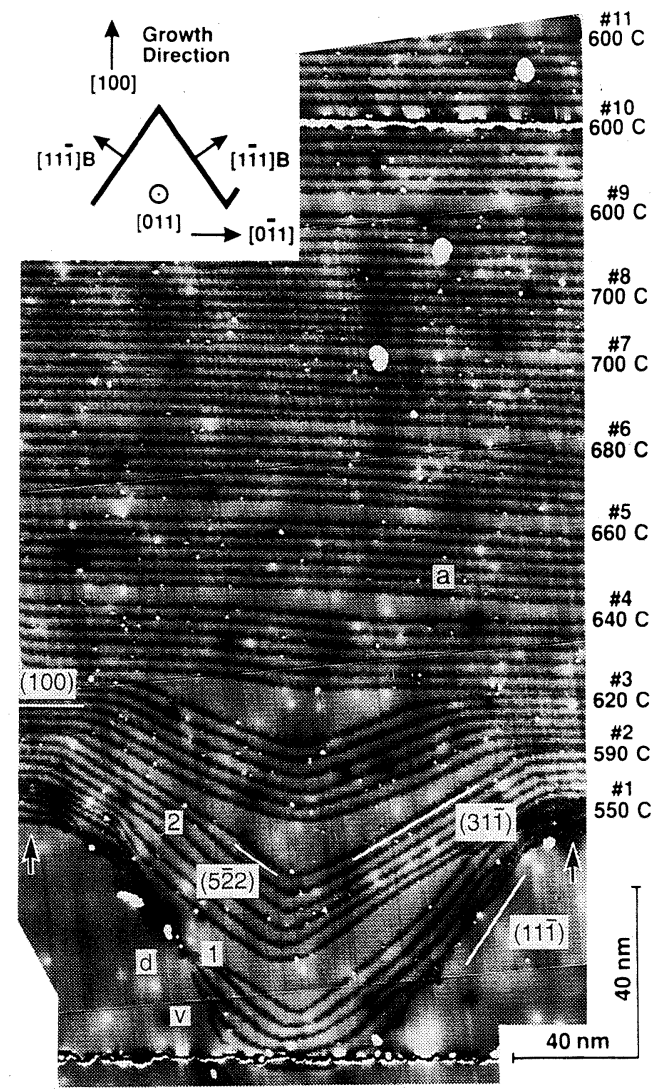

Fig. 6 Montage of STM filled-state images of a (110)-cleaved stack of Gas quantum well wires imbedded in a (AlAs) $)_{4} /(\mathrm{GaAs})_{8}$ superlattice (The temperatures indicated on the right-hand side are the growth temperatures). The images were acquired at a sample bias voltage of $-2.3 \mathrm{~V}$ and a tunneling current of $50 \mathrm{pA}$. The AlAs layers appear darker than the surrounding GaAs layers. The white protrusions about $25 \AA$ in diameter (labeled 'd') represent electrically active $\mathrm{Be}_{\mathrm{Ga}}$ dopants in the top several layers. Atomically localized black and white dots are cleavage-induced vacancy ' $v$ ' or adatom 'a', respectively. [From Ref. 40)]

Smith et al. ${ }^{39)}$ using XSTM. Their results (shown in Fig. 5) have demonstrated that the interface roughness of $(\mathrm{AlGa}) \mathrm{As} / \mathrm{GaAs}$ grown without the interruption technique is around 4-5 atomic layers for the "inverted" interface and 2-3 atomic layers for the "normal" interface. On the other hand, the $(\mathrm{AlAs})_{2} /(\mathrm{GaAs})_{4}$ superlattice grown with growth interruption shows much sharper interfaces (Fig. 5(c)). The extent of the interface roughness derived from this XSTM work is consistent with the XSTS study results (Sec. 4) but larger than the XTEM and photoluminescence results where the structural information is obtained by averaging over a large number of unit cells.

Shown in Fig. 6 is a montage of XSTM filledstate images of $(\mathrm{AlAs})_{4}(\mathrm{GaAs})_{8} / \mathrm{GaAs}$ crescentshaped quantum wires (QWRs) obtained recently by Pfister $e t a l .{ }^{40)}$ The sample used in this study was grown by MBE at several growth temperatures on a prepatterned GaAs (100) substrate. Holographic lithography and wet etching techniques were applied for the preparation of the $190 \mathrm{~nm}$ periodicity $\mathrm{V}$-groove patterning. The orientation of the Vgroove sidewalls in this sample is close to that of the (111) B planes as shown in the figure. Substrate temperatures ranging from 550 to $700^{\circ} \mathrm{C}$ were used during MBE growth to investigate the growth temperature effects. The direct growth of crescentshaped QWR on a V-groove substrate is based on the differences in the surface migration properties of the cation atoms on the different atomic planes during the growth. In most cases the surface cation atoms migrate more quickly on the higher-index planes. This usually leads to a higher growth rate of GaAs on higher-index planes. As can be seen in this image, the GaAs layers grown on the (311) plane are thicker than the (100)-oriented layers and the $(5 \overline{2} 2)$-oriented layers are thicker than the (311)oriented layers. Besides the growth-rate differences, the doping behavior in GaAs grown on the high-index surfaces by MBE is generally much more complicated than that in layers grown on (100)-oriented substrates. In this study, the authors were also able to observe single electrically active dopant atoms. This opens a new avenue for studying incoporation mechanisms of dopants grown along unconventional orientations.

\section{XSTS of (AIGa)As/GaAs Heterostructures}

The most important parameters in tailoring the electronic and photonic properties of semiconductor heterostructures are the conduction and valence band discontinuities between the host materials and the whole band profiles across the heterostructures. Since many novel device ideas require operating these heterostructures based on the quantum principle, the dimensions of these quantum heterostructures are in the nanometer regime. Therefore, it is very important to develop a technique for the direct mapping of the electronic structure across heterostructures with high spatial resolution. Because of its capability to resolve atomic structures in real space and its sensitivity to 
electronic properties of probed materials, XSTM is an excellent candidate for this purpose. Recently, there has been significant progress in this field. We will review the XSTS results in the (AlGa) As/ GaAs system.

It has been found that at the relaxed III-V (110) surface (naturally cleaved surface), the surface-associated states (anion- and cation-like states) move respectively toward the valence and the conduction bands, and as a result the associated mid-gap density of surface states (on the unrelaxed surface) vanishes. Because of this, the relaxed III-V (110) surface is unpinned, or in the other words, the surface Fermi level is not pinned down near the midgap. As a result, the band-edge positions as measured by tunneling spectroscopy on the cleaved III-V surfaces can directly correspond to the bandedge positions within the bulk.

In the past, it was considered that the major advantage of performing XSTS band mapping on III-V heterostructure surfaces was their unpinned nature. However, the studies of Salemink et al. ${ }^{41)}$ revealed that the tip-induced band bending imposes a serious constraint on precisely determining the band edge positions. The tip-induced band bending is particularly important in the case of wide band-gap semiconductors such as GaAs and (AlGa)As since the applied electric field is large during data acquisition. In the case of homogeneous GaAs, since the degree of tip-induced band bending depends on the doping level, this effect can be reduced by simply using heavily doped samples. Unfortunately, for heterojunction or $p-n$ homojuction samples, there are carrier-depleted regions right at the junctions of interest. Thus, the tup-induced band bending effect is an inevitable problem when studying unpinned III-V heterostructure surfaces. Ironically, the solution to this band-bending dilemma is to create a uniformly pinned surface since the uniform extrinsic surface states (no particular localized feature within the band gap) which pin the Fermi levels also screen out the electric field induced by the applied electric field between tip and sample. It was recently found that the sulfide passivation process produces a uniformly pinned cross-sectional surface, ideal for XSTS studies of heterojunctions. ${ }^{3,42-45)}$

Shown in Figs. 7 and 8 are the tunneling spectroscopy results obtained by current imaging tunneling spectroscopy (CITS). In this method, a sample-and-hold controller is applied at every pixel to acquire $I-V$ tunneling spectra at constant tip-sample separation. The tunneling data are then displayed using a normalized conductance $d(\ln I)$
$/ d V=(d I / d V) / I$ versus position method, where the differential conductance $(d I / d V)$ is obtained in this case by numerically differentiating the $I-V$ spectra. The method of using normalized conductance to analyze the spectroscopy data was first proposed by Feenstra $e t a l .{ }^{24)}$ In their approach, $d I / d V$ was obtained by applying the lock-in technique using a small modulation signal with frequency well above the response of the STM feedback system on the bias voltage.

Normalized conductance, which is independent of tip-sample separation, is usually used for identification of density of states features in the STS results. However, when the applied bias voltage is near the band edge position, it can also be directly used to represent the valence or conduction (dependent on the bias polarity) edges relative to the Fermi level. ${ }^{43)}$ This can be understood when we consider that the tunneling current, in the simplest approximation, can be expressed as

$$
I \propto \int_{e V_{0}}^{e V} \exp (-2 \kappa S) \rho_{\mathrm{s}}(E) d E,
$$

where $S$ is the tip-sample separation, $\kappa$ is the decay constant, $V$ is the bias voltage, $V_{0}$ is the band threshold, and $\rho_{\mathrm{s}}(E)$ is the density of states of the sample at the energy $E$. In this approximation, we assume that the density of tip electronic states $\rho_{\mathrm{t}}(E)$ is essentially constant. When $V \approx V_{0}$, we can use the parabolic band approximation for $\rho_{\mathrm{s}}(E)$; therefore, $\rho_{s}(E) \propto\left(E-e V_{0}\right)^{1 / 2}$. At the lowest order, the tunneling current $I$ is then proportional to exp $(-2 \kappa S)\left(V-V_{0}\right)^{3 / 2}$ (in general, $\kappa$ is also bias voltage dependent; however, within a small bias window, we approximate it as a constant).

In this approximation, the normalized conductance,

$$
d(\ln I) / d V=(d I / d V) / I \propto 1 /\left(V-V_{0}\right),
$$

is a quantity independent of the tip-sample separation and diverges as $V \rightarrow V_{0}$. Thus, it can be used as a very sensitive indicator as the bias voltage approaches the band threshold positions. The spatial dependence of the valence band maximum (VBM) can then be estimated directly from the normalized conductance plot since $V_{0}=V-\alpha[d(\ln I)$ $/ d(V)]^{-1}$. In Fig. 7(a), we can see that $d(\ln I)$ $/ d(V)$ at a constant negative sample bias is much larger in the $\left(\mathrm{Al}_{0.3} \mathrm{Ga}_{0.7}\right) \mathrm{As}$ region, indicating the effect of the valence-band offset (VBO). Figure 7(b) shows the resulting valence band plot derived from Fig. 7(a) in the $\left(\mathrm{Al}_{0.3} \mathrm{Ga}_{0.7}\right) \mathrm{As} / \mathrm{GaAs}$ region. From this plot, we can then determine both the VBO value and the electronic effect of asymmetric inter- 
(a)

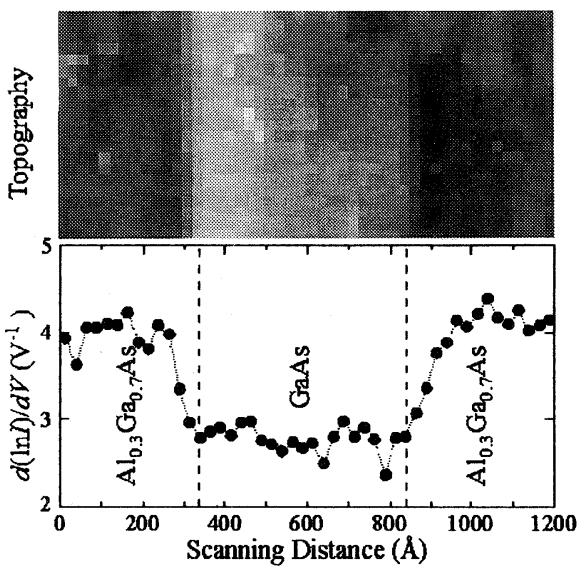

(b)

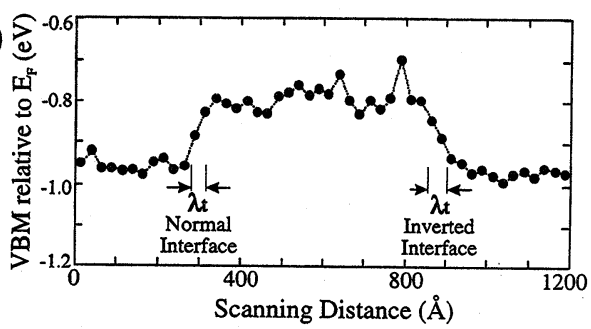

Fig. 7 (a) A 48 pixel $\times 21$ pixel STM image in which tunneling $I-V$ spectra were taken at each pixel in the image. The lower part is the plot of normalized conductance $d(\ln I) / d V$ (numerically differentiated from tunneling $I-V$ spectra) vs position at a negative sample bias $(-1.59 \mathrm{~V})$. Each data point in this plot represents the average result from the $I-V$ spectra along the y direction. (b) the positions of valence-band edges (referenced to the Fermi level) vs position and the transition widths of normal and inverted interfaces. This plot is derived from the normalized conductance plot shown in (a). [From Ref. 3)]

facial roughness. ${ }^{3)}$ The degree of interfacial asymmetry determined from the normalized conductance plot is in excellent agreement with the XSTM images of $\left(\mathrm{Al}_{0.3} \mathrm{Ga}_{0.7}\right) \mathrm{As}$ interfaces grown under similar conditions. ${ }^{39)}$ In Fig. $8(\mathrm{a})$, the $1 /\left(V-V_{0}\right)$ dependence of the $d(\ln I) / d(V)$ can be seen. An experimental $d(\ln I) / d(V)$ curve for GaAs shown in Fig. 8(b) is found to be in good agreement with a theoretical curve of $\alpha /\left(V-V_{0}\right)$ with $\alpha=1.75$ and $V_{0}=-0.6 \mathrm{~V}$. From this example, we can see that the major advantage of using the normalized conductance plot is the capability of efficiently showing the spatial variation of electronic structure

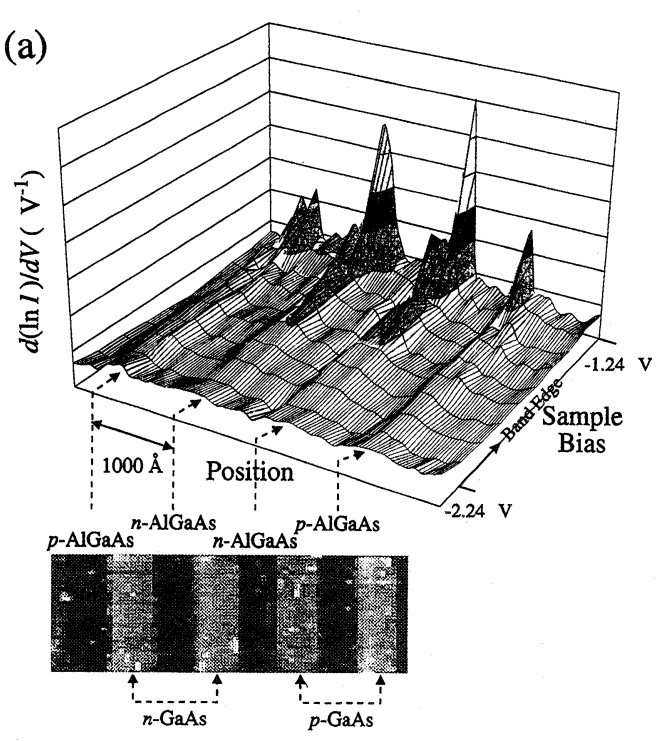

(b)

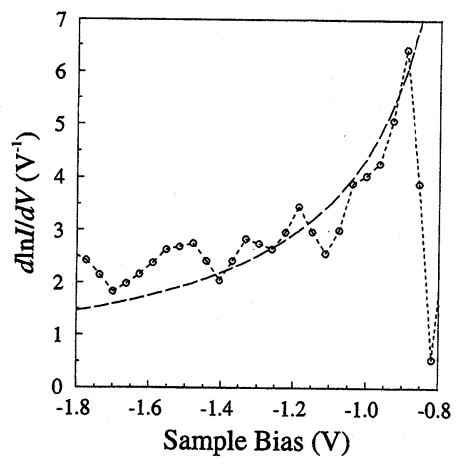

Fig. 8 (a) A plot of normalized conductance vs position and bias over a scanning area of 4500 $\AA \times 1500 \AA$. The corresponding STM image acquired at a sample bias of $-2.35 \mathrm{~V}$ and a tunneling current of $0.3 \mathrm{nA}$ is displayed in the lower left corner. (b) An experimental $d(\ln I) / d V$ curve for GaAs along with a fitting curve of $\alpha /\left(V-V_{0}\right)$ with $\alpha=1.75$ and $V_{0}=-0.6 \mathrm{~V}$. [From Ref. 43)]

since it does not require many fitting parameters and since the exponential dependence of the tipsample separation, which is ubiquitous in imaging the heterostructures, is removed. 


\section{Optical Properties of Semiconductor Heterostructures}

In the preceding sections we described how the XSTM/XSTS technique was applied to obtain some of the most important structural and electronic properties of semiconductor heterostructure systems. Here, we will briefly discuss new methods of exploring the optical properties of semiconductor heterostructures using cross-sectional scanning probe microscopy (XSPM) techniques. Currently, there are two main XSPM methods which have been developed for investigating optical properties of heterostructures with unprecedented spatial resolution. In the first method, the optical properties of heterostructures are investigated by measuring tunneling-induced luminescence (TIL) while the tunneling tip is scanned over the direct-band semiconductor surfaces to obtain STM topographies. ${ }^{46-49)}$ In this method, photons emitted due to electron-hole recombination in direct-band semiconductors are collected by a photodetector combined with a scanning tunneling microscope. The TIL process in direct-band semiconductors can be explained in a simple three-step model: 1 . carrier injection from the tip into the sample, 2 . transport of carriers in the sample (relaxation, diffusion, etc.), and 3. radiative recombination. ${ }^{46}$ Thus, the ultimate spatial resolution is determined by the spatial extension of the second step in this model. Unlike in the conventional cathodoluminescence technique where a high energy electron beam is used, low-energy electrons can be injected by STM at energies of only a few electron volts. As a result, the radiative process is confined to the close vicinity of the injection point. Cross-sectional luminescence maps of multiple-layered ( $\mathrm{AlGa}$ ) As/GaAs heterostructures ${ }^{46-48)}$ and crescentshaped $(\mathrm{AlAs})_{\mathrm{n}}(\mathrm{GaAs})_{\mathrm{m}} / \mathrm{GaAs}$ quantum wires ${ }^{40)}$ obtained by Alvarado et al. showed that spatial resolution down to the nanometer level can be achieved with this method. There is an obvious advantage in using TIL microscopy/spectroscopy. By obtaining atomic resolution STM images simultaneously, the spectral distribution of the emitted photons or the total luminescence intensity measurement can be obtained on any selected regions of an individual quantum structure or on a single adsorbate for detailed optical and chemical-specific information. Very recently, Berndt and Gimzewski also demonstrated that the atomic resolution luminescence map can be obtained by measuring TIL on the reconstructed $\mathrm{Au}(110)$ surface. $^{50)}$ However, in this case, the photons are generated by the inelastic electron tunneling process. ${ }^{51)}$

In the second method, the scanning near-field optical microscope (SNOM) combined with a photodetection system is used for the optical investigation of individual quantum structures..$^{52-54)}$ Two operational modes can be used in the SNOM approach. First, photoluminescence generated by photoexciting the sample with the light passing through the aperture in the SNOM probe can be collected by far-field optics (excitation mode). Alternatively, the sample can be photoexcited by a conventional far-field optical source and the emitted photons transmitted through the aperture in the SNOM probe are locally collected (collection mode). Therefore, the energy range in which luminescence spectra can be obtained by the SNOM/SNOS approach is only limited by the optical excitation source. The results obtained in the low-temperature SNOM/SNOS study of a ( $\mathrm{AlGa})$ As/GaAs cleaved edge overgrowth quantum wire array by Grober and coworkers ${ }^{52,53}$ have demonstrated the usefulness of SNOM/SNOS for investigating spectroscopically individual quantum structures. However, the spatial resolution of this approach is limited by both the aperture diameter of the SNOM probe $(0.25 \mu \mathrm{m}$ in the previous example) and the intrinsic spatial limitation of SNOM.

\section{Summary}

We have reviewed the recent developments of XSTM/XSTS and some related XSPM/XSPS techniques for the structural, electronic, and optical studies of semiconductor heterostructures. Although most of the examples presented here focus on the (AlGa)As / GaAs system because of its importance for optoelectronic and quantum device applications, the XSTM/XSTS technique is readily applicable for the investigation of other material systems. Because of its extremely high sensitivities in analyses of structural and electronic properties, XSTM/XSTS has now become a powerful technique both for imaging low-dimensional structures such as quantum wells and quantum wires and for performing spectroscopic investigations on individual quantum structures.

\section{Acknowledgement}

The authors thank Prof. C. K. Shih at the University of Texas at Austin and Dr. H. W. M. Salemink at IBM Research Divivison-Zurich Research Laboratory for providing figure illustrations. One of us (S.G.) woul like to thank Prof. C. K. Shih for his guidance at the University of Texas at Austin. Last but not least, we thank Dr. S. F. 
Alvarado and Dr. L. A. Nagahara for valuable discussions regarding optical investigations of quantum structures using XSPM approaches. This work was partly supported by the New Energy and Industrial Technology Development organization (NEDO).

\section{Reerences}

1) G. Binnig, H. Rohrer, Ch. Gerber and E. Weibel: Phys. Rev. lett. 49, 57-60 (1982).

2) O. Albrektsen,, D. J. Arent, H. P. Meier and H. W. M. Salemink: Appl. Phys. Lett. 57, 31-33 (1990).

3) S. Gwo, K.-J. Chao, C. K. Shih, K. Sadra and B. G. Streetman: Phys. Rev. Lett. 71, 1883-1886 (1993).

4) T. Kato, F. Osaka and I. Tanaka: Jpn. J. Appl. Phys. 28, 1050-1053 (1989).

5) J. W. Lyding: private communication.

6) J. F. Zheng, J. D. Walker, M. Salmeron and E. R. Weber: Phys. Rev. Lett. 72, 2414-2416 (1994); Erratum, Phys. Rev. Lett. 73, 368 (1994).

7) R. M. Feenstra, D. A. Collins, D. Z.-Y. Ting, M. W. Wang and T. C. McGill: Phys. Rev. Lett. 72, 2749-2752 (1994).

8) A. Y. Lew, E. T. Yu, D. H. Chow: and R. H. Miles: Appl. Phys. Lett. 65, 201-203 (1994).

9) M. Tanimoto and Y. Nakano: J. Vac. Sci. Technol. A8, 553-556 (1990).

10) E. T. Yu, J.-M. Halbout, A. R. Powell and S. S. Iyer: Appl. Phys. Lett. 61, 3166-3168 (1992).

11) M. A. Herman and H. Sitter: Molecular Beam Epitaxy, Springer-Verlag, 120-214 (1989).

12) A. Ourmazd, D. W. Taylor, J. Cunningham and C. W. Tu: Phys. Rev. Lett. 62, 933-936 (1989).

13) H. W. M. Salemink, M. B. Johnson and O. Albrektsen: J. Vac. Sci. Technol. B 12, 362-368 (1994).

14) J. W. Lyding, S. Skala, J. S. Hubacek, R. Brockenbrough and G. Gammie: Rev. Sci. Instrum. 59, 18971902 (1988).

15) Ch. Renner, Ph. Niedermann, A. D. Kent and $\varnothing$. Fischer: Rev. Sci. Instrum. 61, 965-967 (1990).

16) A. R. Smith, S. Gwo and C. K. Shih: Rev. Sci. Instrum. 65, 3216-3219 (1994).

17) R. M. Feenstra, J. A. Stroscio, J. Tersoff and A. P. Fein: Phys. Rev. Lett. 58, 1192-1195 (1987).

18) I. Tanaka, T. Kato, S. Ohkouchi and F. Osaka: J. Vac. Sci. Technol. A8, 567-570 (1990).

19) T. Kato, F. Osaka, I. Tanaka and S. Ohkouchi: J. Vac. Sci. Technol. B9, 1981-1984 (1991).

20) T. Kato and F. Osaka: L1586-L1586 (1991).

21) T. Kato and F. Osaka: J. Appl. Phys. 72, 57165720 (1992).

22) L. Esaki: Physica Scripta T42, 102-109 (1992).

23) R. M. Feenstra, D. A. Collins, D. Z.-Y. Ting, M.
W. Wang and T. C. McGill: J. Vac. Sci. Technol. B 12, 2592-2597 (1994).

24) R. M. Feenstra, A. Vaterlaus, E. T. Yu, P. D. Kirchner, C. L. Lin, J. M. Woodall and G. D. Pettit: in Semiconductor interfaces at the subnanometer scale, NATO ASI Series E No 243, edited by H. W. M. Salemink and M. D. Pashley, 127-137 (Kluwer Press, Dordrecht, 1993).

25) S. Gwo, A. R. Smith, C. K. Shih, K. Sadra and B. G. Streetma: Appl. Phys. Lett. 61, 1104-1106 (1992).

26) W. F. Tseng, J. A. Dagata, R. M. Silver, J. Fu and J. R. Lowney: J. Vac. Sci. Technol. B 12, 373-377 (1994).

27) M. B. Johnson, H. P. Meier and H. W. M. Salemink: Appl. Phys. Lett. 63, 3636-3638 (1993).

28) S. Hosaka, S. Hosoki, K. Takata, K. Horiuchi and N. Natsuaki: Appl. Phys. Lett. 53, 487-489 (1988).

29) S. Kodić, E. J. van Loenen, D. Dijkkamp, A. J. Hoeven and H. K. Moraal: J. Vac. Sci. Technol. A 8, 549-552 (1990).

30) M. B. Johnson, U. Maier, H.-P. Meier and H. W. M. Salemink: Appl. Phys. Lett. 63, 1273-1275 (1993).

31) H. W. M. Salemink and O. Albrektsen: Phys. Rev. B 47, 16044-16047 (1993).

32) R. M. Feenstra, J. M. Woodall and G. D. Pettit: Phys. Rev. Lett. 71, 1176-1179 (1993).

33) R. M. Feenstra, A. Vaterlaus, J. M. Woodall and G. D. Pettit: Appl. Phys. Lett. 63, 2528-2530 (1993).

34) J. F. Zheng, X. Liu, N. Newman, E. R. Weber, D. F. Ogletree and M. Salmeron: Phys. Rev. Lett. 72, 1490-1493 (1994).

35) M. B. Johnson, O. Albrektsen, R. M. Feenstra and H. W. M. Salemink: Appl. Phys. Lett. 63, 2923-2925 (1993).

36) J. F. Zheng, M. Salmeron and E. R. Weber: Appl. Phys. Lett. 64, 1836-1838 (1994).

37) S. B. Ogale, A. Madhukar, F. Voillot, M. Thomsen, W. C. Tang, T. C. Lee, J. Y. Kim and P. Chen: Phys. Rev. B 36, 16620-1672 (1987).

38) B. A. Joyce, P. J. Dobson, J. H. Neave, K. Woodbridge, J. Zhang, P. K. Larsen and B. Bôlger: Surf. Sci. 168, 423-438 (1986).

39) A. R. Smith, K.-J. Chao, C. K. Shih, Y. C. Shih and B. G. Streetman: Appl. Phys. Lett. 4, 478-490 (1995).

40) M. Pfister, M. B. Johnson, S. F. Alvarado, H. W. M. Salemink, U. Marti, D. Martin, F. Morier-Genoud and F. K. Reinhart: Appl. Phys. Lett. 65, 1168-1170 (1994).

41) H. W. M. Salemink, O. Albrektsen and P. Koenraad: Phys. Rev. B 45, 6946-6949 (1992).

42) S. Gwo, K.-J. Chao, A. R. Smith, C. K. Shih, K. Sadra and B. G. Streetman: J. Vac. Sci. Technol. B 11, 1509-1513 (1993). 
43) S. Gwo, A. R. Smith, K.-J. Chao, C. K. Shih, K. Sadra and B. G. Streetman: J. Vac. Sci. Technol. A 12, 2005-2008 (1994).

44) S. Gwo, K.-J. Chao and C. K. Shih: Appl. Phys. Lett. 64, 493-495 (1994).

45) A. R. Smith, S. Gwo, K. Sadra, Y. C. Shih, B. G. Streetman and C. K. Shih: J. Vac. Sci. Technol. B 12, 2610-2615 (1994).

46) P. Renaud and S. F. Alvarado: Phys. Rev. B 4, 6340-6343 (1991).

47) S. F. Alvarado, Ph. Renaud, D. L. Abraham, Ch. Schönenberger, D. J. Arent and H. P. Meier: J. Vac. Sci. Technol. B 9, 409-413 (1991).

48) D. L. Abraham, A. Veider, Ch. Schönenberger, H. P. Meier, D. J. Arent and S. F. Alvarado: Appl. Phys. Lett. 56, 1564-1566 (1990).
49) L. Samuelson, A. Gustafsson, J. Lindahl, L. Montelius, M.-E. Pistol, J.-O. Malm, G. Vermeire and P. Demeester: J. Vac. Sci. Technol. B 12, 25212526 (1994).

50) R. Berndt and J. K. Gimzewski: Phys. Rev. B 48, 4746-4754 (1993).

51) R. Berndt, R. Gaisch, W. D. Schneider, J. K. Gimzewski, B. Reihl, R. R. Schlittler and m. Tschudy: Phys. Rev. Lett. 74, 102-105 (1994).

52) R. D. Grober, T. D. Harris, J. K. Trautman, E. Betzig, W. Wegscheider, L. Pfeiffer and K. West: appl. Phys. Lett. 64, 1421-1423 (1994).

53) R. D. Grober, T. D. Harris, J. K. Trautman and E. Betzig: Rev. Sci. Instrum. 65, 626-631 (1994).

54) H. F. Hess, E. Betzig, T. D. Harris, L. N. Pfeiffer and K. W. West: Science 264, 1740-1745 (1994). 